\title{
Floppy eyelid syndrome
}

\author{
ALEKSANDAR PARUNOVIĆ \\ From the University Eye Clinic, Belgrade, Yugoslavia
}

SUMMARY The results of clinical examinations and follow-up of 7 patients with floppy eyelid syndrome are presented. The most prominent features of the syndrome are floppy upper eyelids that can be easily everted, with papillary conjunctivitis and punctate keratopathy. The ocular changes seem to be the result of an underlying metabolic (or endocrine) disorder. In the patient with the longest follow-up period (10 years) considerable loss of body weight resulted in a dramatic relief of ocular symptoms.

The floppy eyelid syndrome was first reported in 1981 by Culbertson and Ostler. ${ }^{1}$ They considered this syndrome to be a unique clinical entity that had not been previously described. According to them the main characteristics of the disease are floppy upper eyelids and papillary conjunctivitis of the upper palpebral conjunctiva. Culbertson and Ostler considered that in these patients the upper eyelid everts during sleep, resulting in irritation and papillary conjunctivitis. The origin of the syndrome remains unknown.

We report here the findings in 7 patients with floppy eyelid syndrome.

\section{Case reports}

We have seen and followed up 7 patients during 1972-81. Most of them had the clinical findings as described by Culbertson and Ostler ${ }^{1}$ but some differed in the clinical picture or in the course of the disease.

\section{CASE 1}

We saw this patient for the first time in 1972 . He was then a 44-year-old man, extremely overweight (Fig. 1) but otherwise healthy. No member of his family has had a similar disease. Ten years prior to our examination he had developed burning, itching, and marked redness of his eyes. Symptomatic treatment had been of no help. The skin of his upper eyelids was slightly swollen, and here and there a scanty mucous exudate was present. The entire conjunctiva was hyperaemic, with extensive papillary hypertrophy of the upper palpebral conjunctiva. The surface of the

Correspondence to Dr A. Parunović, Oćna Klinika, Višegradska 22. YU-11000, Belgrade, Yugoslavia. cornea was covered with coarse epithelial infiltrates, most of which stained. The cornea was more affected in its upper part, and a small, slowly progressive

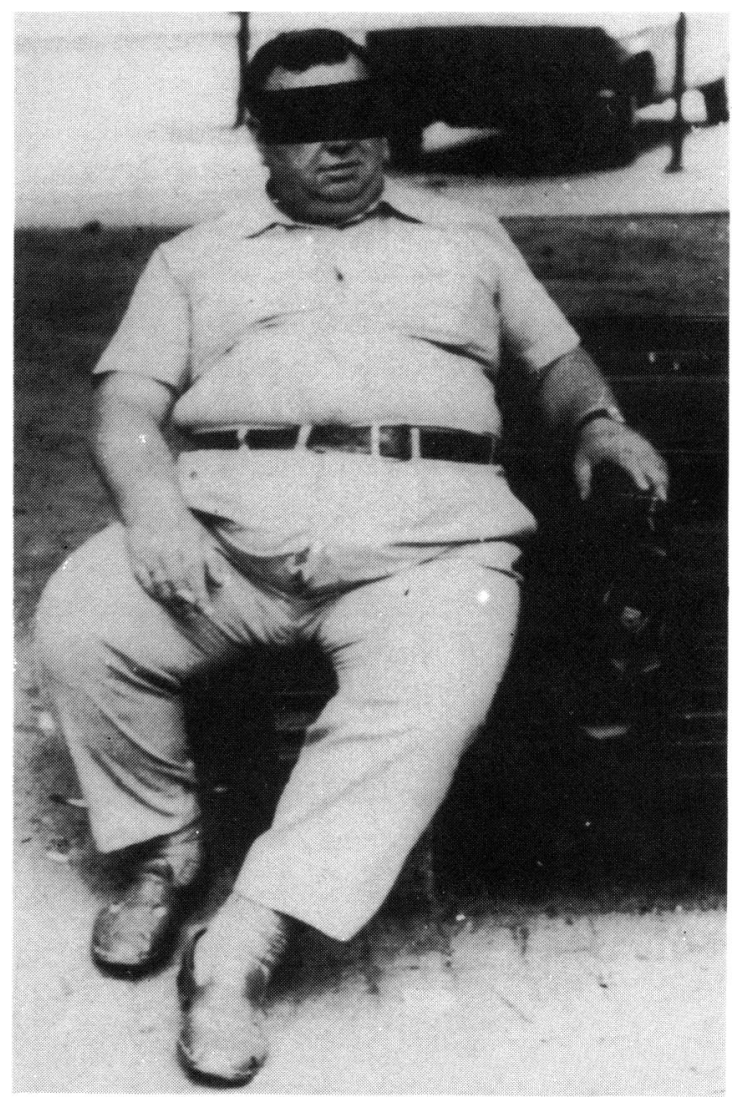

Fig. 1 Obesity in floppy eyelid syndrome. 
pannus was also present at the upper limbus. The most unusual feature of the clinical picture was the upper lids. Their motility was normal but they seemed to have no elasticity at all. The eyelid could be everted by simply pulling its skin upwards. The ocular changes were bilateral and symmetrical.

In 1972 he told us that the condition of his eyes had remained unchanged for years. Innumerable specimens for bacteriological, virological, and cytological examinations were collected but no micro-organisms or abnormal cells were found. Thorough metabolic end endocrine examinations were performed, but no positive result was obtained. He was referred in 1974 to Moorfields Eye Hospital in London, where he was thoroughly examined and discharged as keratoconjunctivitis of unknown origin, probably the result of a metabolic disorder. The condition of both eyes remained the same until 1978. At that time the patient managed to cut down his body weight considerably by following a strict diet; he lost as much as $30 \mathrm{~kg}$. His eye disease promptly and dramatically improved. In 3 months the irritation disappeared, there were only $2-3$ staining points on the cornea, and the bulbar conjunctiva became quiet for the first time in 15 years. We discontinued all treatment, and there has been no relapse of the disease in the past 5 years. He has no symptoms of irritation and is very satisfied. However, the most characteristic sign of the disease, floppy lids, remained unchanged.

\section{CASE 2}

We saw this 47 -year-old man in 1981. His eye troubles began 2 years before with redness, burning, and slight discharge. Both eyes are affected, but he noticed the condition of the left eye to be worse (Fig. 2). The right upper eyelid could be everted in the normal way, the left upper eyelid was floppy and easily everted. The palpebral and bulbar conjunctivae were injected, and

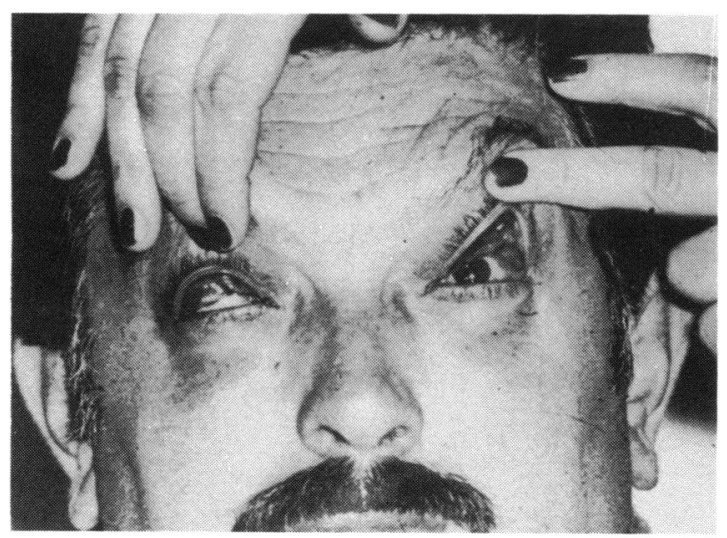

Fig. 2 Unilateral case. there were large papillae on the upper palpebral conjunctiva. There was also a fine punctate staining of the corneal epithelium. The lesions were bilateral but more pronounced in the left eye.

Following the suggestion of L. K. Schwartz et al. (personal communication) we advised this patient to have his eyelids taped shut during the night. After a month he informed us that his wife had noticed his left eye to be slightly open during the night but the upper eyelid had not been everted. The taping of the eyelids neither changed the condition of his eyes nor lessened his troubles.

The patient's general health was good except that he was grossly overweight. Endocrine, metabolic, and dermatological examinations did not reveal any abnormality.

CASE 3

This 53-year-old man was first seen in 1979. He was extremely overweight. The skin of his face was so floppy that dermatologists suspected-but could not prove-a cutis laxa syndrome. He had had continuous eye symptoms for at least 7 years. The visual acuity of his left eye was decreased, LE 6/18. Both upper eyelids could be everted and pulled up all the way to the eyebrow with almost no resistance (Fig. 3). The palpebral conjunctiva was injected and covered by large papillae. The bulbar conjunctiva was also injected. There were coarse, punctate staining defects of the corneal epithelium all over the cornea. The changes were symmetrical and bilateral. The visual acuity of the left eye was lowered owing to the presence of a typical first-stage keratoconus.

\section{Discussion}

Floppy lid syndrome (or, as one should call it, floppy lid keratoconjunctivitis) is a distinct clinical entity of unknown origin. The most prominent and unique symptom is floppy, rubbery upper eyelids which can be everted easily and pulled very high up. Papillary

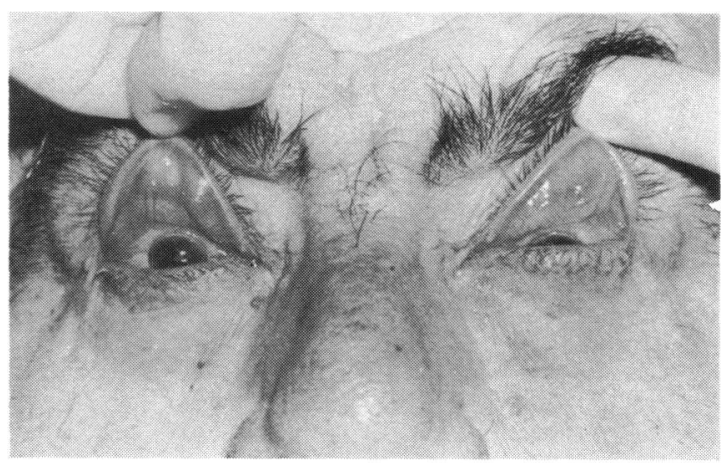

Fig. 3 Upper eyelids are easily everted. 
hypertrophy of the upper palpebral conjunctiva and coarse epithelial keratitis are also present. It has yet to be confirmed whether conjunctival and corneal changes are due to eversion of the upper eyelid during sleep, as suggested by Culbertson and Ostler. ${ }^{1}$ As noted above, 5 out of 7 of our patients tried taping their eyes shut during the night. Three of them had distinct relief of subjective troubles from this measure but the corneo-conjunctival lesions changed only slightly (case 2).

Case 3 is notable for the association of floppy lid syndrome with keratoconus. A similar association has already been reported ${ }^{2}$ so it may not be a simple coincidence.

Another point remains obscure. If corneoconjunctival lesions are the result of nocturnal lagophthalmos why are the corneal changes not localised in the lower half of the cornea, as has been reported $^{3}$ ? In all our 7 patients the corneal lesions were uniformly distributed over the cornea. If there was a difference, then it was the upper part of the cornea which was more affected.

The patient with the longest follow-up (case 1) is particularly interesting. During the 18 years of his illness he has never had either nocturnal lagophthalmos or night eyelid eversion, yet his corneo-conjunctival changes have been among the most prominent in the entire group. Finally, his corneo-conjunctival lesions almost disappeared after his metabolism was changed. Furthermore, in the patient having unilateral floppy eyelid, corneal and conjunctival changes were present in both eyes. It is likely that upper eyelid eversion during the night might aggravate pre-existing keratoconjunctivitis, but it also seems unlikely that the everted eyelid results in corneal and conjunctival lesions.

We subjected all our 7 patients to extensive endocrine, metabolic, and dermatological examinations, but the results were of no interest. The results of bacteriological, virological, and cytologic examinations were as reported by Culbertson and Ostler.'

\section{References}

1 Culbertson WW, Ostler HB. The Floppy eyelid syndrome. Am J Ophthalmol 1981; 92: 568-75.

2 Ocular Microbiology and Microbiology Group Meeting. Am J Ophthalmol 1982; 93: 249.

3 Katz J, Kaufman HE. Corneal exposure during sleep (nocturnal lagophthalmos). Arch Ophthalmol 1977; 95: 449-53. 